\title{
pyramus and thisbe: FGF genes that pattern the mesoderm of Drosophila embryos
}

\author{
Angelike Stathopoulos, ${ }^{1}$ Bergin Tam, ${ }^{1}$ Matthew Ronshaugen, ${ }^{1}$ Manfred Frasch, ${ }^{2}$ \\ and Michael Levine ${ }^{1,3}$ \\ ${ }^{1}$ Department of Molecular and Cell Biology, Division of Genetics \& Development, University of California, \\ Berkeley, California 94720-3204, USA; ${ }^{2}$ Brookdale Department of Molecular Cell and Developmental Biology, \\ Mount Sinai School of Medicine, New York, New York 10029, USA
}

The Heartless (Htl) FGF receptor is required for the differentiation of a variety of mesodermal tissues in the Drosophila embryo, yet its ligand is not known. Here we identify two new FGF genes, thisbe (ths) and pyramus (pyr), which probably encode the elusive ligands for this receptor. The two genes exhibit dynamic patterns of expression in epithelial tissues adjacent to Htl-expressing mesoderm derivatives, including the neurogenic ectoderm, stomadeum, and hindgut. Embryos that lack ths ${ }^{+}$and $p^{\prime} r^{+}$exhibit defects related to those seen in $h t l$ mutants, including delayed mesodermal migration during gastrulation and a loss of cardiac tissues and hindgut musculature. The misexpression of Ths in wild-type and mutant embryos suggests that FGF signaling is required for both cell migration and the transcriptional induction of cardiac gene expression. The characterization of $h t l$ and ths regulatory DNAs indicates that high levels of the maternal Dorsal gradient directly activate $h t l$ expression, whereas low levels activate ths. It is therefore possible to describe FGF signaling and other aspects of gastrulation as a direct manifestation of discrete threshold readouts of the Dorsal gradient.

[Keywords: FGF; heartless receptor; dorsal; Drosophila embryo; pyramus; thisbe]

Received October 31, 2003; revised version accepted February 13, 2004.

FGF signaling molecules have been implicated in both the movement and specification of different mesoderm lineages in vertebrate embryos (Szebenyi and Fallon 1999; Tickle and Munsterberg 2001). For example, FGF4 and FGF8 are required for the invagination of epiblasts into the primitive streak of mouse embryos, whereas FGF8 and FGF24 appear to specify the posterior mesoderm cells in zebrafish embryos (Sun et al. 1999; Draper et al. 2003). Given the large number of FGF signaling molecules in vertebrates, it is often difficult to distinguish between disruptions in movement or specification. It is conceivable that FGF signaling controls both processes.

Invertebrate embryos offer an opportunity to investigate this issue because they contain relatively few FGFs. The nematode worm Caenorhabditis elegans contains two FGF ligands, Egl-17 and Let-756. Mutants lacking egl-17 $7^{+}$activity exhibit defects in the migration of the sex myoblasts to the gonad and in the differentiation of the muscle cells that surround the developing vulva (Burdine et al. 1998). Thus, it would appear that FGF signaling is required for both movement and specifica-

${ }^{3}$ Corresponding author.

E-MAIL mlevine@uclink.berkeley.edu; FAX (510) 643-5785.

Article and publication are at http://www.genesdev.org/cgi/doi/10.1101/ $\operatorname{gad} .1166404$ tion. Mutants lacking Let-756 display developmental arrest early in larval stages, but the exact cause of this defect has not been determined (Roubin et al. 1999).

Previously, the only FGF gene known in Drosophila was branchless, which is essential for the morphogenesis of the trachea, air sacs, and male genital imaginal disc (Sutherland et al. 1996; Ahmad and Baker 2002; Sato and Kornberg 2002). During embryogenesis, branchless is expressed in a highly dynamic fashion in discrete epithelial cells of developing embryos. The Branchless ligand triggers the activation of the Breathless FGF receptor and thereby controls the movement (branching) of the trachea (Sutherland et al. 1996). It is conceivable that this Branchless-Breathless interaction is also important for the specification of at least a subset of the tracheal cell types. Branchless does not appear to influence the specification or movement of mesoderm lineages during earlier periods of Drosophila embryogenesis.

Although only one FGF ligand has been identified, Drosophila contains two FGF receptors, Breathless and Heartless (Htl). The Htl receptor is essential for the development of various mesoderm lineages, including cardiac tissues, hindgut visceral musculature, and the body wall muscles (Beiman et al. 1996; Gisselbrecht et al. 1996; San Martin and Bate 2001). Htl is initially expressed throughout the mesoderm of early embryos (Shishido et al. 1997), and its activation is thought to 
trigger the spreading of the mesoderm across the internal surface of the neurogenic ectoderm. The mesoderm cells that come into contact with the dorsal ectoderm receive an inductive signal, Dpp, which triggers the expression of genes such as tinman (tin) and even-skipped (eve) that are required for the differentiation of cardiac and pericardial tissues, respectively (Staehling-Hampton et al. 1994; Frasch 1995). However, the mechanism of Htl activation is uncertain (for review, see Wilson and Leptin 2000). It has been suggested that localized FGFs emanating from the neurogenic ectoderm might be responsible for $\mathrm{Htl}$ activation and provide an instructive cue that guides the migration of the mesoderm. An alternative view is that $\mathrm{Htl}$ plays a permissive role in migration by rendering the mesoderm competent to respond to an unknown localized signal.

$\mathrm{Htl}$ may be required both for the spreading of the mesoderm and the subsequent specification of cardiac tissues. The misexpression of Dpp throughout the ectoderm, in both dorsal and ventral regions, causes widespread activation of tin expression within the mesoderm (Frasch 1995). However, eve expression is not expanded, and it has been suggested that its activation depends on both Dpp signaling (normally achieved through spreading) and a second dorsally localized signal, possibly FGF (Frasch 1995; Carmena et al. 1998; Michelson et al. 1998b; Halfon et al. 2000). The analysis of the hindgut visceral musculature provides evidence for this dual role of FGF signaling in movement and specification (San Martin and Bate 2001). The activation of $\mathrm{Htl}$ is required for the initial spreading of the visceral mesoderm around the hindgut, as well as the subsequent differentiation of the hindgut musculature.

To investigate the function of FGF signaling in the early embryo, we identified the Htl ligands, which have eluded intensive genetic screens. The present study identified two closely linked genes, thisbe (ths) and pyramus $(p y r)$, which encode FGF signaling molecules that appear to function in a partially redundant fashion to activate Htl. Ths and Pyr are most closely related to the FGF8/ $17 / 18$ subfamily, which controls gastrulation as well as heart and limb development in vertebrates (e.g., Maruoka et al. 1998; Sun et al. 1999; Reifers et al. 2000). Both ths and pyr are expressed in the neurogenic ectoderm during the spreading of the internal mesoderm in gastrulating embryos. These two genes also exhibit dynamic expression in the stomadeum, hindgut, and muscle attachment sites of older embryos. These sites of expression closely match the genetic function of $h t l$ described in previous studies. Moreover, a small deletion that removes both ths and pyr causes a variety of patterning defects, including delayed spreading of the mesoderm during gastrulation, the loss of cardiac tissues and hindgut visceral musculature, and abnormal patterning of the body wall muscles. These defects are similar to those seen for htl mutants. The ectopic expression of Ths in the early mesoderm of gastrulating embryos causes an expansion in the domain of $\mathrm{Htl}$ activation and a corresponding expansion in the eve expression pattern. These observations suggest that $\mathrm{Htl}$ controls both the spreading of the mesoderm and (along with Dpp and Wingless) the specification of pericardial cells. Computational methods were used to identify a mesoderm-specific enhancer for $h t l$ that is directly activated by peak levels of the maternal Dorsal gradient. Because ths is directly activated by low levels of the gradient, it is possible to describe gastrulation as a direct manifestation of discrete threshold readouts of the Dorsal gradient.

\section{Results}

The Identification of two linked FGF genes, thisbe (ths) and pyramus (pyr)

A recent microarray screen identified a gene called Neu4 (CG12443) that is directly activated by low levels of the maternal Dorsal gradient in broad lateral stripes that encompass the entire presumptive neurogenic ectoderm (Stathopoulos et al. 2002). Computational methods were used to identify a tissue-specific enhancer that directs this Neu4 expression pattern. The enhancer contains three tightly linked, high-affinity Dorsal-binding sites. It was found to map quite far from the predicted Neu4 promoter, $\sim 16 \mathrm{~kb} 5^{\prime}$ of the coding region. To confirm this remote linkage of the Neu4 enhancer, rapid amplification of cDNA ends (RACE) was done using embryonic RNA to identify $5^{\prime}$ Neu 4 coding sequence. These assays identified three short exons that map far $5^{\prime}$ of the previously identified coding sequences (Fig. 1A). The newly identified exons place the Neut enhancer within intron 2 of the transcription unit and not in the $5^{\prime}$ regulatory region. The new exons were found to share homology with another predicted open reading frame (ORF), CG13194, that maps $\sim 80 \mathrm{~kb} 5^{\prime}$ of the Neu4 gene. RACE assays were done to identify the 5' CG13194 coding sequences. We hereafter refer to Neu4/CG12443 as thisbe (ths) and the second, related gene CG13194 as pyramus (pyr; summarized in Fig. 1D), for the "heartbroken" lovers described in Ovid's Metamorphoses because the genes are linked and the mutant phenotype exhibits a lack of heart (see below).

Ths and Pyr are related to FGF8/17/18 signaling molecules

The previously identified CG12443 and CG13194 ORFs predicted by the Berkeley Drosophila Genome Project (BDGP) do not share obvious homology with genes of known function. However, the newly identified ths and pyr $5^{\prime}$-exons encode peptides related to FGF ligands (Fig. 1B,C). FGF molecules are highly divergent and exhibit limited amino acid sequence identity (Szebenyi and Fallon 1999; Ornitz 2000). The core FGF domain is composed of $12 \beta$ strands separated by coiled-coil regions that form a trefoil structure. The most highly conserved amino acid residues between the Ths and Pyr FGF domains, and other FGFs including FGF8 and Branchless, tend to map in regions that are essential for the integrity of the structure, particularly the $\beta$ strands (Fig. 1B). An- 
A

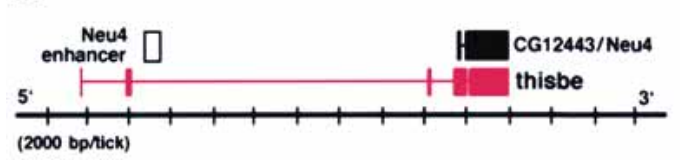

c

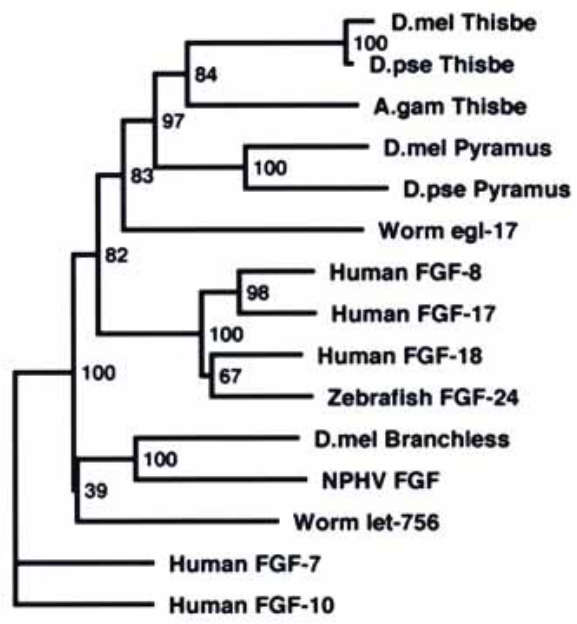

B

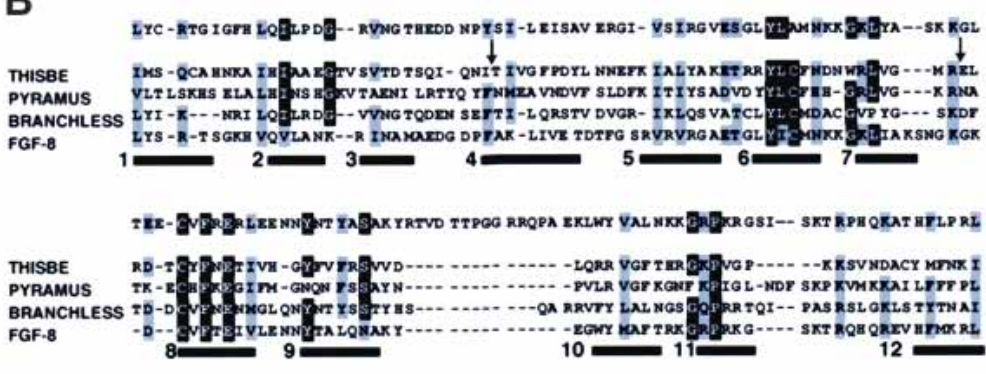

D

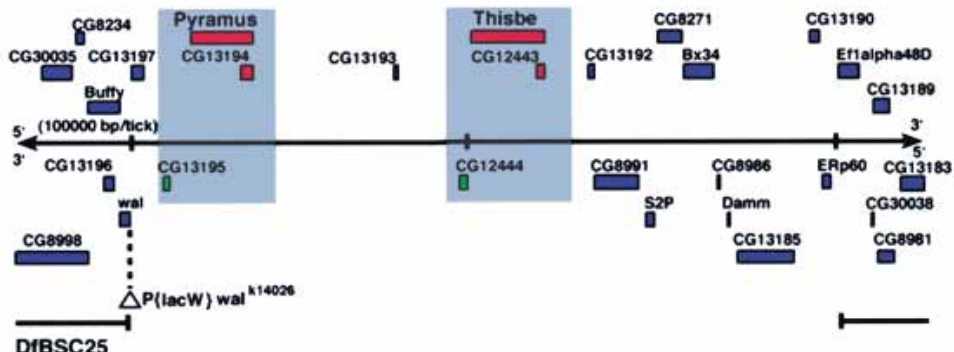

Figure 1. Thisbe and Pyramus are FGF-ligands. (A) RACE was done to isolate the $5^{\prime}$ sequence of the CG12443/Neu4 open reading frame (black) predicted by BDGP. Three previously unidentified exons were found that now place the Neut enhancer (open box) in the second intron of the thisbe gene (red). (B) An alignment of the putative protein sequences of $D$. melanogaster Thisbe and Pyramus with D. melanogaster Branchless, Human FGF8, and the PFAM Hidden Markov Model (HMM) consensus for the FGF protein family (top line). The HMM consensus represents the residues having the highest probability of occurrence for each position in an alignment of all known FGF proteins (Bateman et al. 2002). Dark highlighting indicates positions where $80 \%$ of proteins are identical; light highlighting indicates positions where $80 \%$ of the residues are similar using a PAM 250 substitution matrix. Intron positions are indicated with arrows. Black bars beneath the alignment indicate the positions of the $12 \beta$ strands in the FGF trefoil structure. (C) Reconstruction of phylogenetic relationships between the Drosophila FGF proteins Thisbe and Pyramus with other FGF proteins from fly, worm, fish, mosquito, virus, and human. Branch lengths are proportional to sequence divergence. The numbers at nodes are bootstrap values and indicate degree of support for particular branching relationships on a 1:100 scale. (A.gam) Anopheles gambiae; (D.mel) Drosophila melanogaster; (D.pse) Drosophila pseudoobscura; (Zebrafish) Danio rerio; (Human) Homo sapiens; (NPHV) Bombyx mori nuclear polyhedrosis virus. See Materials and Methods for accession numbers. $(D)$ Genomic organization of pyramus and thisbe. pyr and ths (formerly CG13194 and CG12443, respectively) are linked genes separated by 20 kb. CG13195 and CG12444 (green boxes) encode related proteins that contain periplasmic binding domains. The light-blue shading indicates the proposed limits of a gene duplication event that resulted in these duplicated pairs of genes. Df(2R)BSC25 is a deficiency that has $\sim 200 \mathrm{~kb}$ removed, including the $\sim 110-\mathrm{kb}$ interval containing the pyr and ths genes. The deletion also removes a number of flanking genes, including the Damm caspase. To date, none of these have been implicated in embryonic development. The horizontal lines at the bottom of the diagram indicate the flanking regions that are retained in the deficiency chromosome.

other notable feature of the Ths and Pyr proteins is that they are both predicted to contain $\mathrm{N}$-terminal signal sequences, suggesting that the proteins are present extracellularly (Nielsen et al. 1997). They also lack several of the signature amino acid residues that have been implicated in the interaction of certain FGFs with heparin sulfate proteoglycans (HSPGs; Ornitz 2000). Because FGF-HSPG interactions are thought to mediate sustained activation of FGF receptors, it is conceivable that Ths and Pyr function as transient signals, which is consistent with their dynamic patterns of expression during development (see below).

Phylogenetic analyses were performed using the neighbor-joining method (Fig. 1C). The principal finding is that among all known FGFs, Ths and Pyr are most closely related to EGL-17 in C. elegans and the FGF8/ 17/18 subfamily in vertebrates (Burdine et al. 1997; Maruoka et al. 1998). In contrast, Branchless (Bnl) and Let-
756, the only other nonchordate FGFs, are most closely related to viral FGF-like molecules (Sutherland et al. 1996). Both ths and pyr are conserved in the Drosophila pseudoobscura (D.pse) genome, but there is only a single gene in the mosquito, Anopheles gambiae (A.gam; Fig. 1C). This single FGF gene is more closely related to ths than pyr. It would appear that ancestral dipterans contained a single copy of an FGF gene that underwent duplication after the divergence of mosquito but prior to the divergence of $D$. melanogaster and D. pseudoobscura. Two Drosophila genes, CG13195 and CG12444, that are closely linked to $p y r$ and ths are also related to one another, suggesting that an $\sim 50$-kb interval containing the ancestral FGF gene, along with its neighbor, underwent a tandem duplication event (Fig. 1D, gray boxes). Greater sequence conservation exists between thisbe orthologs (D.mel Thisbe and D.pse Thisbe) than pyramus orthologs (D.mel Pyramus and D.pse Pyramus; 
Stathopoulos et al.

Fig. 1C). This result suggests that selection may be acting on ths to maintain some ancestral function, whereas pyramus has been released from constraint and is rapidly evolving in the Drosophilids.

\section{pyr and ths are dynamically expressed} throughout embryogenesis

In situ hybridization assays were done as a first step toward determining gene function. Initially, the two genes exhibit a very similar expression profile (Fig. 2A,E). During cellularization, each gene is expressed in broad lateral stripes within the neurogenic ectoderm. Staining is excluded from the presumptive mesoderm in ventral regions and from the anterior third of the embryo. This ths pattern is maintained during mesoderm invagination and the rapid phase of germ-band elongation (Fig. 2B,C). In contrast, at the onset of germ-band elongation, the $p y r$ expression pattern is rapidly refined and expression is detected only in dorsal and ventral regions of the neuro- genic ectoderm (Fig. 2F,G). At the completion of elongation, both genes are expressed in discrete regions of the epidermis, including different subsets of the ventral neuroblasts (Fig. 2I,M).

After the completion of germ-band elongation, ths and pyr exhibit dynamic and, in part, distinct patterns of expression in different tissues. For example, pyr is expressed in ectodermal stripes (Fig. $2 \mathrm{H}$ ) that refine to smaller spots, which are adjacent to pericardial cells in the dorsal mesoderm (Fig. 2M,N). ths exhibits similar, but weaker expression (Fig. 2D,I; data not shown), with additional expression in founders of the visceral muscles (Fig. 2J, arrowhead). After germ-band retraction, both genes are expressed in the ectodermal derivatives of the gut, the proctodeum and stomodeum, as well as the central nervous system (Fig. 2K,O) and in the muscle attachment sites at the segment borders (Fig. 2L,P, arrowhead). A recurring theme in the complex expression patterns is the appearance of Ths and Pyr in different epithelial tissues at the time when derivatives of the mesoderm that
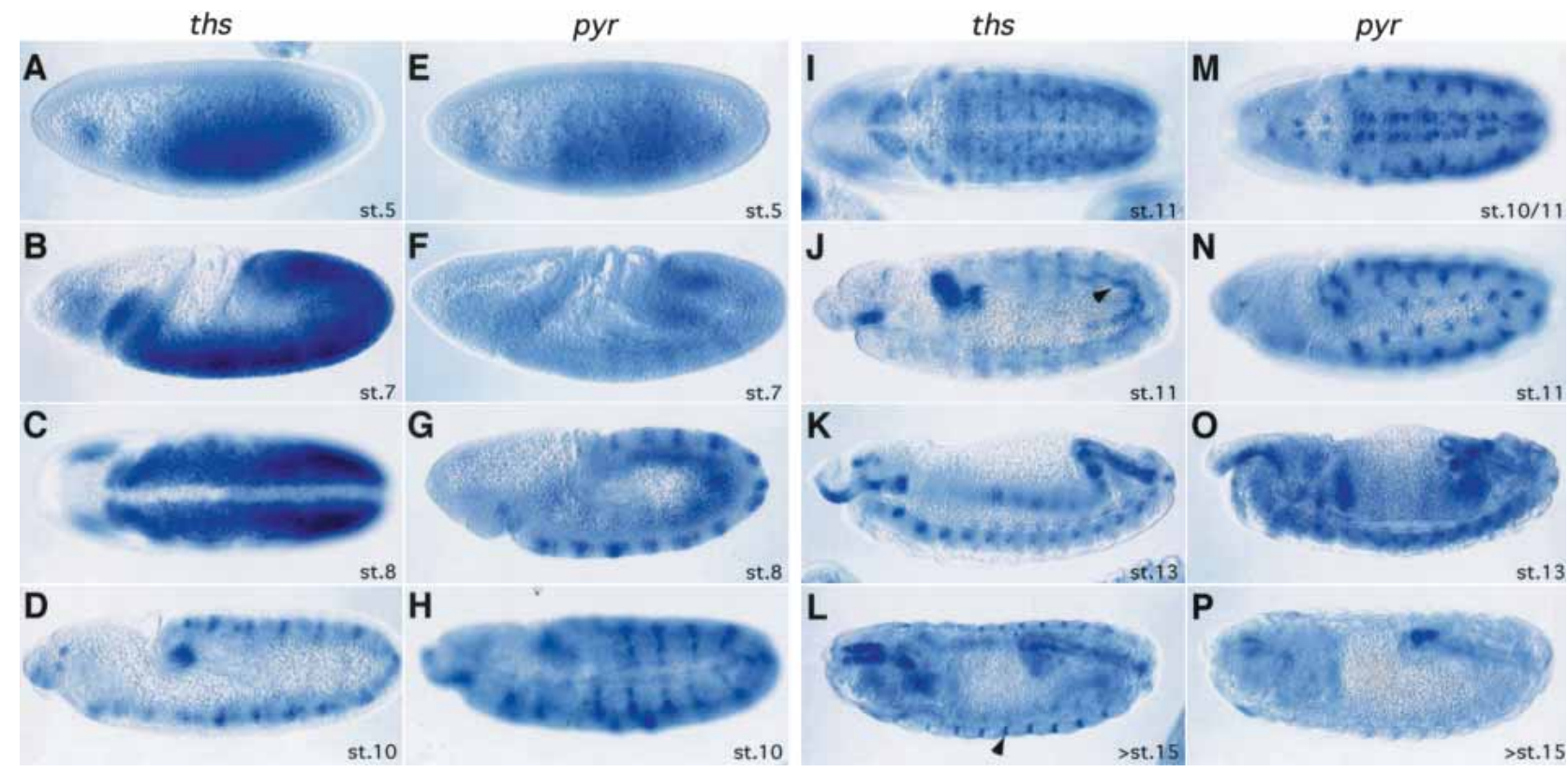

N

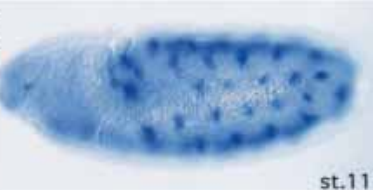

$\mathrm{O}$

$\mathbf{L}$

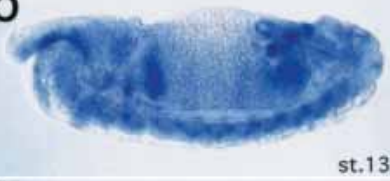

st.11
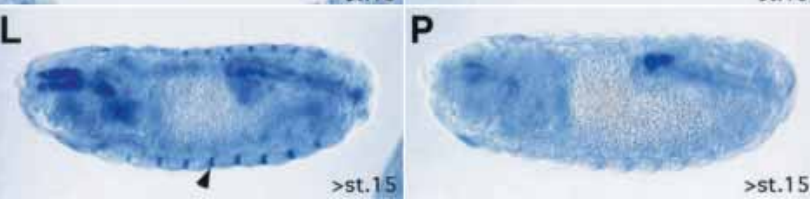

Figure 2. thisbe and pyramus expression patterns throughout embryogenesis. In situ hybridizations depicting ths $(A-D, I-L)$ and $p y r$ $(E-H, M-P)$ localization in the embryo. For all embryos, anterior is to the left. Ventral views are depicted in $C, I$, and $M$. Lateral views are depicted for all other embryos with the dorsal surface on top. $(A, E)$ Views of cellularized embryos (stage 5$)$. Both pyr $(E)$ and ths $(A)$ are expressed in broad lateral stripes. $(B, F)$ Ventral views of gastrulating embryos beginning rapid germ-band elongation (stage 7$)$. pyr expression diminishes $(F)$ while ths remains robust $(B)$. $(C)$ View of an embryo undergoing germ-band elongation (stage 8). ths expression remains strong throughout the neurogenic ectoderm but is excluded from the midline. (I) At later stages, ths expression is restricted to a subset of neuroblasts. In contrast, pyr expression is present only in select regions of the neurogenic ectoderm, specifically the dorsal region $(G)$ and in distinct domains of the ventral region $(M) .(D, H, I, M)$ Lateral and ventral views of embryos that have completed germ-band elongation (stage 10). ths expression becomes limited to the dorsal neurogenic ectoderm $(D)$ and then falls away almost completely from these dorsal ectodermal regions as well, with staining remaining within visceral mesoderm founder cells $(J)$. $(H)$ pyr is expressed in stripes throughout the embryo. $(N)$ Later, pyr is expressed in 11 groups of cells within the ectoderm in proximity to cells of the mesoderm that will take on a pericardial fate. ths is also expressed in stripes and spots similar to pyr, but ths expression in these domains is only apparent after significant overstaining (data not shown). $(K, O)$ Views of embryos undergoing germ-band retraction (stages 13-14). pyr and ths are expressed in regions flanking the proctodeum and stomodeum, the ectodermal derivatives of the gut, as well as the central nervous system. $(L, P)$ Views of late-stage embryos having completed germ-band retraction (stages 15-17). ths and pyr expression remains associated with the foregut and the hindgut. Additional expression of ths can be observed in what appear to be the muscle attachment sites ( $L$; arrowhead). 
express Htl become associated with these tissues (see below).

\section{Embryos lacking ths}

\section{and pyr exhibit gastrulation defects}

The similar early expression profiles of ths and pyr raise the possibility that they function in a redundant fashion to control mesoderm spreading during gastrulation. This would explain why previous genetic screens identified the Htl receptor, but failed to identify the FGF ligands. To date, zygotically lethal mutations have not been identified in either ths or pyr. Perhaps genetic redundancy exists between these two genes such that mutation of both would be required to reveal defects like those seen for htl mutants. To circumvent this potential problem, we identified a small chromosomal deletion Df(2R)BSC252 that removes both genes and was generated by a male-specific recombination event using the P-element $\mathrm{P}\{\mathrm{lacW}\} \mathrm{wal}^{\mathrm{k} 14026}$. The exact breakpoints of this deficiency were defined; it removes $\sim 200 \mathrm{~kb}$ of genomic DNA, including the entire 110-kb interval that contains ths and pyr (Fig. 1D). No more than 18 predicted genes are removed, which is not a very big number considering that $<1 \%$ of all the genes in the Drosophila genome produce specific embryonic patterning defects when disrupted by zygotic mutation (Simpson 1983; Anderson et al. 1985). Indeed, none of the predicted genes, several of which encode components of the apoptosis pathway (Liu et al. 1999; Harvey et al. 2001), have been implicated in the control of embryonic development. Embryos were collected from the deficiency strain, and in situ hybridization assays confirm that ths and pyr are not expressed in mutant embryos that are homozygous for the deletion (data not shown).

In normal embryos, activation of the Htl signaling pathway correlates with the spreading of the mesoderm along the internal surface of the neurogenic ectoderm. This signaling is absent in the mesoderm of htl mutants (Gabay et al. 1997). To determine whether ths;pyr deficiency mutants have similar defects in mesoderm spreading, we analyzed sections of embryos stained with antibodies recognizing either Twist to observe the mesoderm or dpERK to monitor activation of the $\mathrm{Htl}$ pathway. Mesoderm cells begin to migrate at stage 7,8 of embryogenesis (Fig. 3A), and dpERK staining is detected in those cells that have come into contact with the ectoderm (Fig. 3G). In contrast, mesoderm migration is defective in ths; pyr deficiency mutants (Fig. 3, cf. C and A); they also lack dpERK staining (in the mesoderm), as seen in htl mutants (Fig. 3, cf. I and G; Beiman et al. 1996; Gisselbrecht et al. 1996). The mesoderm has completed its migration by early stage 10 of embryogenesis (Fig. 3B). In both wild-type and mutant embryos, the mesoderm comes into contact with Dpp-expressing cells in the dor-
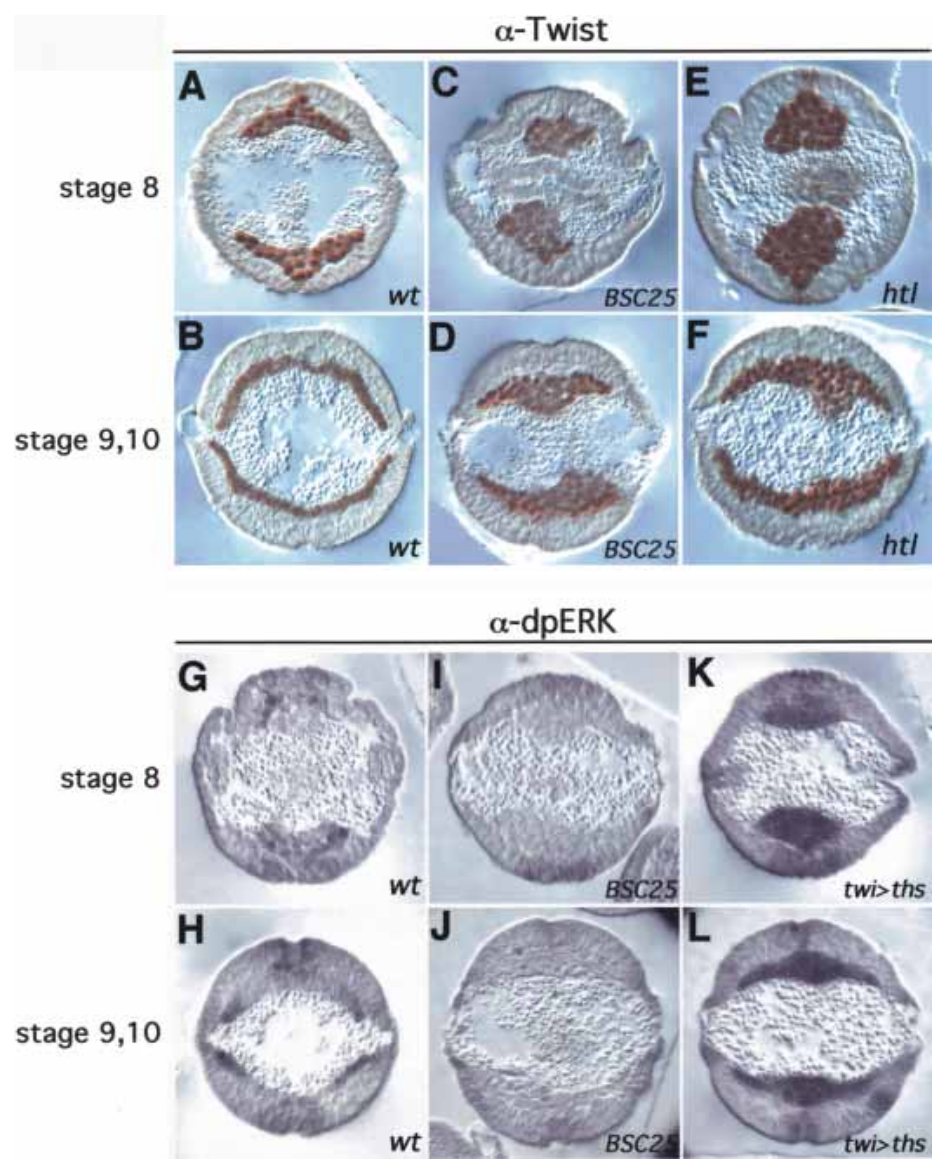

Figure 3. $\mathrm{Df}(2 \mathrm{R}) \mathrm{BSC} 25$ homozygotes exhibit delayed mesoderm spreading that is similar to Htl mutants. Embryos were stained with anti-Twist $(A-F)$ or anti-dpERK antibodies $(G-L)$ and sectioned as described (see Materials and Methods). $(A, B, G, H)$ Wild-type embryos. $(C, D, I, J) D f B S C 25$ mutant embryos. $(E, F$,$) htl mutant embryos. (K, L)$ Embryos misexpressing ths in the mesoderm. Sections were made of embryos either in the early stage of mesoderm spreading, stage $8(A, C, E, G, I, K)$, or once spreading in the wild type is complete, stage $9,10(B, D, F, H, J, L) .(A-F)$ Wild-type embryos with Twist staining showing early mesodermal cells beginning to migrate $(A)$ and once migration is complete at later stages $(B)$. In BSC25 deficiency and $h t 1$ mutant embryos, mesoderm cells remain clumped $(C, E)$ at a stage when wild-type cells have initiated migration $(A)$, but do spread eventually and contact Dpp-expressing ectodermal cells $(D, F)$ almost as fully as wild-type cells do $(B)$. $(G-L)$ dpERK staining in wildtype embryos is present in mesodermal cells in contact with the ectoderm early $(G)$ and is limited later to only the cells at the leading edge of the migrating mesoderm $(H)$. dpERK staining is absent from the mesodermal cells of BSC25 deficiency mutant embryos both early $(I)$ and late $(I)$. When ths is ectopically expressed in the mesoderm using a twi-Gal4 driver (twi $>$ ths), dpERK staining is expanded to all cells of the entire mesoderm at both early $(K)$ and late $(L)$ stages. 
Stathopoulos et al.

sal ectoderm but shows various degrees of multilayered and irregular arrangements. The expanded mesoderm forms a monolayer in wild-type embryos, but displays multiple layers in ths;pyr deficiency mutants (Fig. 3D) and $h t l$ mutants (Fig. 3F). dpERK staining is restricted to the dorsal mesoderm of wild-type embryos at stage 9,10 embryos (Fig. 3H), but is absent in ths;pyr deficiency mutants (Fig. 3J). Staining is expanded in embryos that ectopically express the ths ligand in the mesoderm (Fig. $3 \mathrm{~L})$. These results are consistent with a requirement of the Ths and Pyr ligands for activation of the Htl receptor in mesoderm migration during gastrulation (see Discussion).

\section{Mesoderm defects in ths;pyr deficiency mutants are similar to those seen in htl mutants}

As a result of mesoderm spreading, the dorsal mesoderm comes into contact with the dorsal ectoderm and is induced by a localized Dpp signal to express a variety of regulatory genes required for the differentiation of cardiac tissues and visceral mesoderm (Staehling-Hampton et al. 1994; Frasch 1995). Several marker genes were used to characterize the lethal phenotype caused by the deletion that may result from the inability of dorsal mesoderm lineages to differentiate (Figs. 4, 5). These include eve, tin, bagpipe (bap), and mef2 (Azpiazu and Frasch 1993; Bodmer 1993; Bour et al. 1995; Frasch 1995; Lilly et al. 1995). In htl mutants, there is either a loss or reduction in the expression of each of these regulatory genes. Similar disruptions are observed in ths;pyr (BSC25) defi- ciency homozygotes. There is a complete loss of Eve staining in the pericardial cells and dorsal muscle founder cells of both $h t 1$ and BSC25 deficiency mutants (Fig. 4B,C; cf. 4A), but the central nervous system (CNS) pattern is unaffected (Fig. 4E,F; cf. 4D). After germ-band elongation, tin expression can be seen in two distinct lineages of the dorsal mesoderm, the visceral mesoderm (Fig. 4G, arrow) and cardiac mesoderm (Fig. 4G, arrowhead; Azpiazu and Frasch 1993; Bodmer 1993). There is a severe reduction of tin expression in the cardiac lineage in htl mutants and BSC25 deficiency homozygotes (Fig. $4 \mathrm{H}, \mathrm{I} ; \mathrm{cf} .4 \mathrm{G})$. There is also reduced expression of bap in some segments, although expression is mostly normal (bap is required for visceral mesoderm formation; Fig. $4 \mathrm{~K}, \mathrm{~L}$; cf. 4J). The minor defects in bap expression may result from incomplete spreading of the mesoderm along the entire anterior-posterior axis in $h t l$ and deficiency mutant embryos, whereas the more severe defects in the transcriptional up-regulation of eve and tin may result from a late requirement of $\mathrm{Htl}$ activation after mesoderm spreading (see below).

The mef2 gene is expressed in a variety of mesodermal tissues, including the differentiating body wall muscles and the dorsal vessel, or heart (Fig. 5; Bour et al. 1995; Lilly et al. 1995). Most aspects of this staining pattern are unaffected in BSC25 deficiency homozygotes, but there is a selective loss of staining in the dorsal mesoderm that forms the heart cells and dorsal somatic muscles (Fig. 5, cf. C and A). Similar defects have been described for $h t l$ mutants (Fig. 5B), and in both cases, the selective loss of dorsal mesoderm derivatives can be explained by the

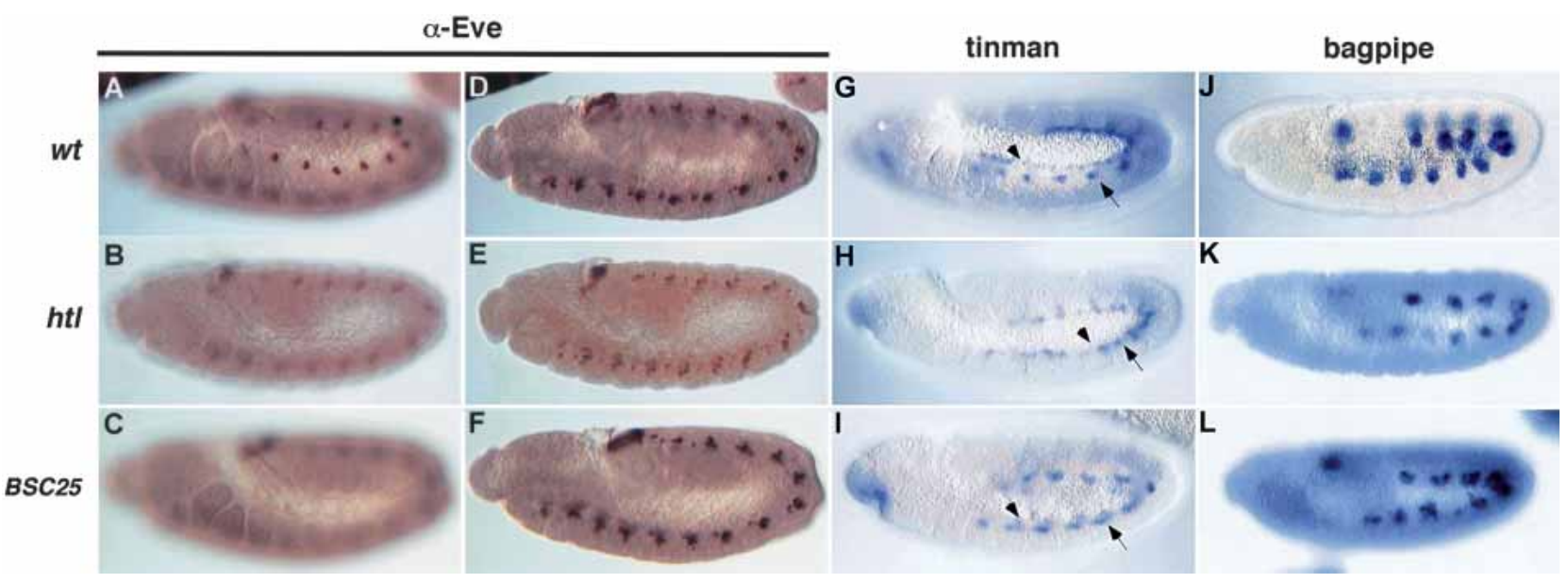

Figure 4. $\mathrm{Df}(2 \mathrm{R}) \mathrm{BSC} 25$ homozygotes exhibit abnormalities similar to those seen in htl mutants. Embryos have undergone germ-band elongation. They are oriented with anterior to the left and dorsal up. $(A, D, G, J)$ Wild-type embryos were stained with the anti-Eve antibody $(A, D)$, tin and bap RNA probes $(G, J$, respectively). (A) Eve is expressed in approximately three pericardial cells within each hemisegment of the dorsal mesoderm. $(D)$ Eve is also expressed in specific subsets of neurons within each neuromere of the developing ventral nerve cord. $(G)$ tin mRNAs are expressed in two lineages of the dorsal mesoderm, the visceral mesoderm (arrow), and the cardiac mesoderm (arrowhead). ( $J$ ) At this same stage, bap mRNA is expressed in a broad domain of the dorsal mesoderm. $(B, E, H, K)$ Mutant embryos homozygous for a null mutation in htl. Eve staining is lost in the pericardial cells $(B)$, but unaffected in the CNS $(E)$. There are reduced levels of tin mRNAs $(H)$ in the cardiac mesoderm (arrowhead), whereas staining in the visceral mesoderm is nearly normal (arrow). bap expression within the visceral mesoderm is also reduced in a subset of segments $(K)$. $(C, F, I, L)$ Mutant embryos homozygous for the BSC25 deficiency that removes the ths and pyr genes. These embryos exhibit phenotypes that are similar to those observed for htl mutants $(B, E, H, K)$. 


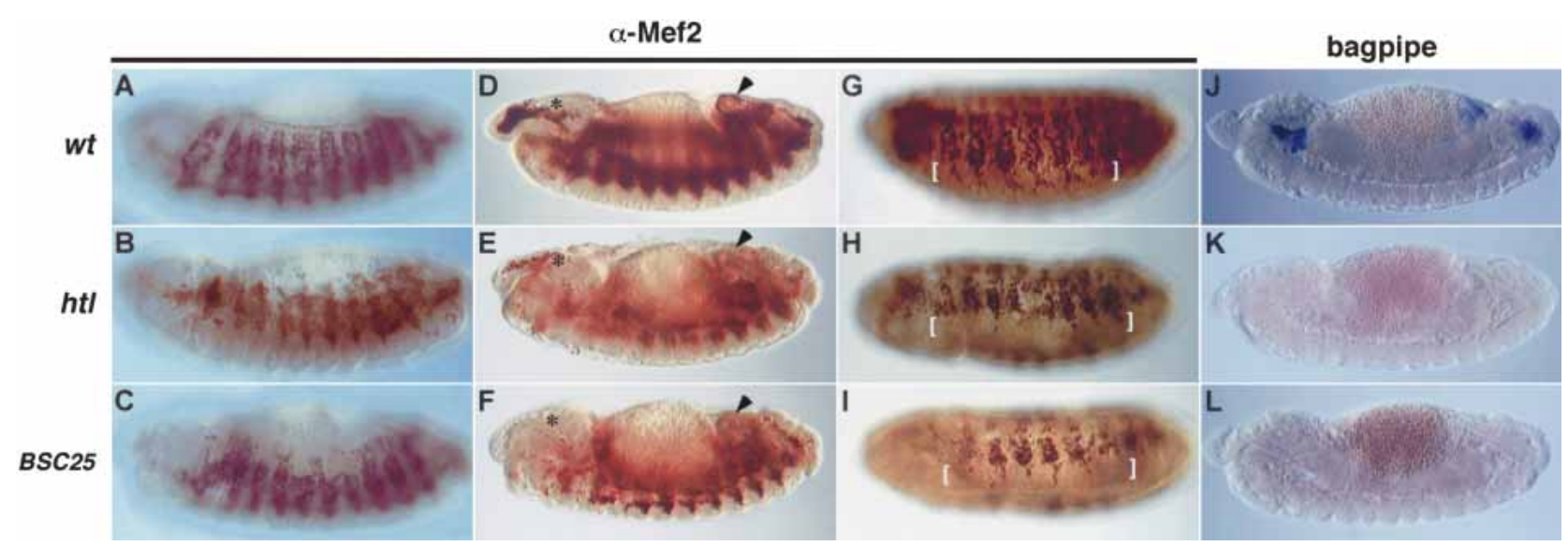

Figure 5. FGF signaling is essential for the differentiation of specific muscle types in advanced-stage embryos. Embryos have undergone germ-band retraction and were stained with either a Mef2 antibody $(A-I)$ or bap mRNA probe $(J-L)$. $(A, D, G, J)$ Wild-type embryos. (A) Mef2 is expressed in a variety of differentiating mesoderm lineages in retracted embryos, including the dorsal vessel (heart) and somatic body wall muscles. Mef2 is also expressed in the differentiating pharyngeal muscles associated with the stomadeum (asterisk in $D$ ), the hindgut musculature (arrowhead in $D$ ), and ventral oblique body wall muscles (white brackets in $G$ ). (J) bap mRNAs are detected in the pharyngeal muscles and hindgut musculature. $(B, E, H, K) h t l$ mutant embryos. $(B)$ Mef 2 staining indicates a loss of dorsal mesoderm derivatives, including the heart and dorsal mesoderm derivatives. (E) Mef2 staining is reduced in the pharynx (asterisk) and absent in the hindgut (arrowhead). $(H)$ There is also a severe reduction in the number of ventral oblique muscles (brackets). (K) bap mRNAs are not expressed in the pharynx or hindgut musculature (cf. $J)$. $(C, F, I, L)$ BSC25 deficiency homozygotes. These embryos exhibit phenotypes that are similar to those observed for htl mutants $(B, E, H, K)$.

combined effects of uneven mesoderm spreading and the loss of late Htl signaling after mesoderm spreading (see Discussion).

A late embryonic requirement for $\mathrm{Htl}$ signaling has been established for the visceral musculature surrounding the hindgut. Hindgut visceral mesoderm (HVM) migrates over the hindgut ectoderm during dorsal closure, and this migration depends on both Wingless $(\mathrm{Wg})$ and $\mathrm{Htl}$ (San Martin and Bate 2001). Unlike the migration of the HVM, subsequent differentiation does not require Wg signaling, but depends solely on Htl (San Martin and Bate 2001). In htl and BSC25 deficiency mutants, Mef2 staining is absent from the hindgut, implying that the hindgut musculature has not differentiated (arrowheads, Fig. 5E,F; cf. 5D). Although a role for Htl in the differentiation of the pharyngeal muscles has not been previously described, Mef2 staining of pharyngeal muscle is reduced in $h t l$ mutants (asterisk, Fig. 5E; cf. 5D) with an even more severe reduction observed in BSC25 deficiency embryos (asterisk, Fig. 5F; cf. 5D). In addition, the visceral mesoderm associated with both the stomadeum and hindgut in these advanced-stage embryos also expresses bap (Fig. 5J). This staining is lost in $h t l$ and BSC25 deficiency mutant embryos (Fig. 5K,L). Both ths and pyr are expressed in the stomadeum and hindgut at the time when these mesodermal derivatives form, and thus it seems likely that the encoded FGFs function as signals to control their movement and/or differentiation at these later stages of embryogenesis.

A dominant-negative form of $\mathrm{Htl}$ has been shown to block the formation of the muscle founder cells and the differentiation of the ventral oblique muscles (Gajewski et al. 1999). Most ventral oblique muscles are absent in $h t l$ and BSC25 deficiency mutant embryos (brackets, Fig.
$5 \mathrm{H}$, I; cf. 5G). This observation is consistent with the model that the localized expression of Pyr and Ths in the ventral ectoderm, possibly within ventral neuroblasts, is required for the specification of muscle founder cells through activation of the Htl receptor.

\section{Ectopic expression of Ths mimics constitutive activation of the $\mathrm{Htl}$ receptor}

If Ths and Pyr are the ligands for Htl, then the misexpression of one or both genes in the mesoderm should be sufficient to trigger its activation. A full-length ths cDNA was misexpressed in the mesoderm using a twistGal4 transgene (Fig. 6A,B). The levels of ectopic ths mRNAs are several-fold higher than the endogenous products (Fig. 6A, arrowheads), but nonetheless the mesoderm still spreads toward the dorsal ectoderm (Fig. 3L). There is a substantial increase in the number of Eveexpressing mesodermal cells in embryos ectopically expressing ths in the mesoderm (Fig. 6C,D; cf. 4A or 6J). A similar expansion is obtained with constitutively activated forms of the $\mathrm{Htl}$ receptor or Ras kinase (Halfon et al. 2000) or when ths is expressed using an ectodermal driver, 69B-gal4 (Fig. 6M). These results are consistent with Ths acting as a ligand for the Htl receptor.

Mef2 staining was also examined in embryos that misexpress ths throughout the mesoderm. These embryos exhibit no obvious defects in most mesoderm derivatives such as the hindgut musculature with this marker (Fig. $6 \mathrm{E}$, arrowhead), although the ventral oblique muscles are either absent or unable to extend into ventral regions (Fig. 6F, brackets). One possible explanation is that guidance of the ventral oblique muscles is controlled by pyr and/or ths expression in ventral neuro- 
Stathopoulos et al.

Figure 6. Misexpression of Ths causes a variety of mesoderm patterning defects. Embryos were stained using a ths riboprobe $(A, B)$, an anti-Eve antibody $(C, D, G-$ $O)$, or the anti-Mef2 antibody $(E, F) .(A, B)$ ths misexpression in the mesoderm using a twi-Gal4 driver and UAS-ths transgene. ths expression was detected by in situ hybridization and is misexpressed in the ventral mesoderm in cellularizing embryos (data not shown) and during the formation of the ventral furrow $(A)$. Weaker staining can be seen in the neurogenic ectoderm (arrowheads in $A$ ), which corresponds to the expression of the endogenous gene. ths mRNAs persist in the mesoderm during elongation and shortening ( $B$; data not shown). ( $C, D)$ Eve staining in embryos that overexpress ths using the twi-Gal4 driver. An expansion in the number of Eve-expressing pericardial cells is already detected at the completion of germ-band elongation, when the cardiac mesoderm is first induced (data not shown). Expanded Eve staining persists during germ-band shortening $(C)$ and after the completion of germ-band retraction $(D) .(E, F)$ Mef2 staining in embryos that ectopically express ths using the twi-Gal4 driver. Staining is essentially normal during elongation (data not shown), but pat-

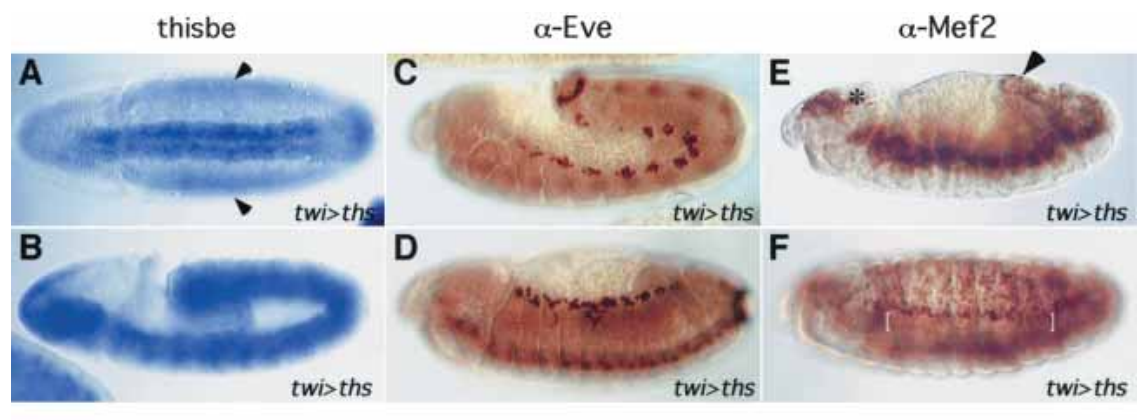

terning defects can be seen at the completion of germ-band retraction $(E, F)$. There is an expansion in the number of Mef2-expressing cells in dorsal mesoderm derivatives $(E)$, and a block in the formation of ventral oblique muscles (brackets, $F)$. Despite these abnormalities, most mesoderm lineages are formed, including the pharyngeal muscles (asterisk in $E$ ) and hindgut musculature (arrowhead in $F)$. $(G-O)$ Embryos were stained using antibodies against Eve and $\beta$-gal to distinguish mutant embryos from wild-type embryos, those carrying the $f t z$-lac $Z$ balancer chromosome. Anti- $\beta$-gal staining in broad stripes corresponding to where $f t z$ drives expression of lac $Z$ can be observed in $J$ and $M$, but this staining is absent from mutant embryos that do not contain the balancer chromosome $(G-I, K, L, N, O)$. $(G-I)$ Different forms of the Htl receptor were expressed in the mesoderm using twist-Gal4. The embryo in $G$ is homozygous for the BSC25 deficiency and expresses a wild-type Htl transgene (UAS-Htl) in the mesoderm (twi > Htl). It lacks expression in the pericardial cells, but exhibits normal staining in the developing CNS. The embryos in $H$ and $I$ are also homozygous for the deficiency, but express the activated Htl receptor (UAS-actHtl) in the mesoderm (twi $>$ actHt1). Eve staining is partially restored in the pericardial cells during germ-band shortening (arrows in $H$ ) and after retraction is complete $(I)$. (J) Expression of Eve in a wild-type background is limited to about three cells per hemisegment. Broad stripes of expression can be seen that correspond to expression of $\beta$-gal by the $f t z$-lac $Z$ transgene from the balancer chromosome. $(K, L)$. Thisbe was expressed in the mesoderm of embryos homozygous for the BSC25 deficiency using the twist-Gal4 driver (BSC25; twi $>$ ths). Eve expression is partially restored in the pericardial cells during germ-band shortening $(K)$ and after retraction is complete $(L) .(M-O)$ Thisbe was expressed in the ectoderm of embryos using the 69B-Gal4 driver $(69 \mathrm{~B}>$ ths $)$. (M) In wild-type embryos, a substantial expansion of pericardial cell specification is observed. When Thisbe is ectopically expressed in the ectoderm of embryos homozygous for the BSC25 deficiency using 69B-Gal4 (BSC25; 69B-Gal4), Eve expression is restored in the pericardial cells in a more uniform domain along the anterior-posterior axis during both germ-band shortening $(N)$ and after retraction is complete $(O)$.

blasts and that ectopic expression of ths at high levels within the mesoderm masks this guidance mechanism (see Discussion).

\section{Genetic complementation}

of ths;pyr deficiency mutants

The simplest model for the mutant phenotype seen in deficiency homozygotes is that the loss of Ths and Pyr blocks the activation of the $\mathrm{Htl}$ receptor, which in turn causes impaired spreading and induction of the mesoderm. To test this model, a genetic complementation experiment was done using a constitutively activated form of the $\mathrm{Htl}$ receptor that in theory no longer requires ligand binding for activation (Michelson et al. 1998a). The mutant receptor was expressed in the developing mesoderm of transgenic embryos using a twist-Gal4 transgene (Greig and Akam 1993). Mutant embryos homozygous for the ths; pyr deficiency never exhibit any eve expression in developing pericardial cells (e.g., Fig. 4C). However, introduction of the activated $\mathrm{Htl}$ receptor partially restores Eve expression in the dorsal mesoderm (Fig. 6H,I, arrows). Staining tends to be stronger in posterior segments, but some of the embryos exhibit Eve 
staining in anterior regions as well. Similar overexpression of the wild-type $h t l$ does not rescue the pericardial Eve expression pattern in mutant embryos (Fig. 6G). These results indicate that the constitutively activated Htl receptor partially circumvents the need for Ths and Pyr in the differentiation of the dorsal mesoderm.

We also tested whether ths expression was sufficient to complement the BSC25 deficiency. When ths is expressed in the mesoderm there is strong, but somewhat erratic rescue of the pericardial cells (Fig. 6K,L). There is more uniform rescue when ths is expressed in the ectoderm using a 69B-Gal4 driver (Fig. 6M-O). These results agree with our findings that mesoderm spreading and induction are normally coordinated by FGF signals emanating from the ectoderm (see Discussion).

\section{FGF signaling components are direct targets of the Dorsal gradient}

Previous studies have shown that ths is directly activated by low levels of the Dorsal gradient in the neuroectoderm (Stathopoulos et al. 2002). ths is probably kept off in the ventral mesoderm by the localized Snail re- pressor because the neuroectoderm enhancer contains an optimal Snail-binding site. It is conceivable that the Dorsal gradient controls mesoderm spreading by differentially regulating the ths/pyr FGF ligands in the neuroectoderm and the FGF receptor and intracellular signaling components in the mesoderm.

To investigate this possibility we used computational methods to identify putative Dorsal target enhancers for

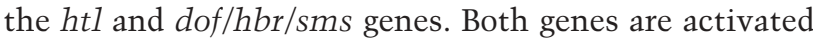
in the presumptive mesoderm prior to the formation of the ventral furrow, and both are required for the spreading of the mesoderm after invagination (Beiman et al. 1996; Gisselbrecht et al. 1996; Michelson et al. 1998a; Vincent et al. 1998; Imam et al. 1999). A survey of the $h t 1$ locus identified a cluster of two putative Dorsal-binding sites and two copies of a distinct sequence motif, CA CATGT, which probably binds the Twist activator and is found in several Dorsal target enhancers (Stathopoulos et al. 2002). The Dorsal-Twist binding cluster is located within the first intron of the htl gene (Fig. 7A). When expressed in transgenic embryos, this 800-bp fragment directs lac $Z$ expression in the ventral furrow and invaginated mesoderm (Fig. 7B-D). A putative dof/hbr/sms
A
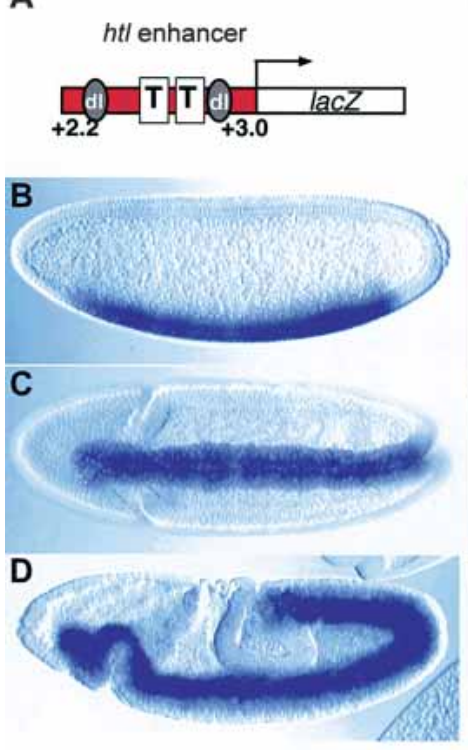

$\mathbf{E}$
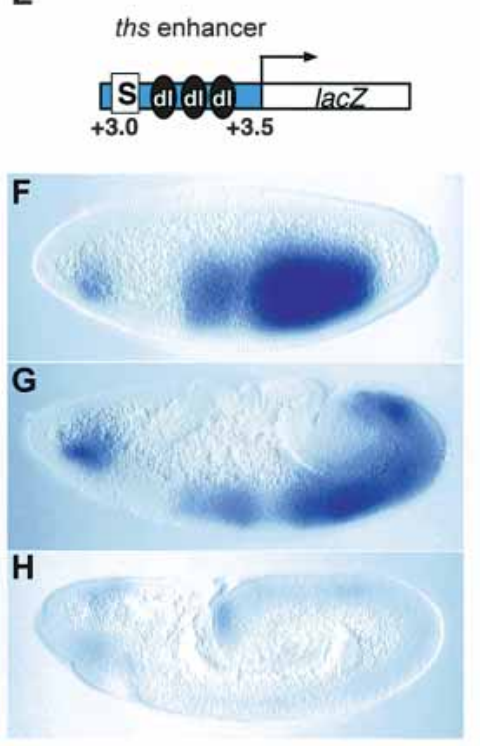

I
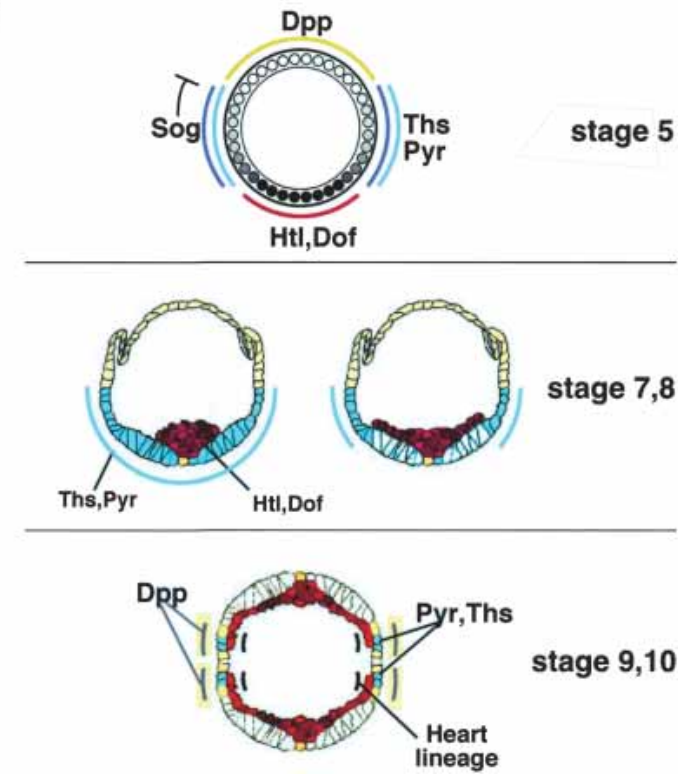

stage 5

stage 9,10

Figure 7. FGF signaling activation is a direct readout of Dorsal threshold outputs. A mesoderm enhancer was identified within the $h t l$ gene $(A)$. The previously identified ths enhancer is shown in $E$. The $h t l$ enhancer is $800 \mathrm{bp}$, whereas the ths enhancer is $500 \mathrm{bp}$. Putative Dorsal-binding sites are indicated in the diagrams (dl), as well as additional sequence motifs, including Twist E-box recognition sequences $(\mathrm{T})$ in the $h t 1$ enhancer $(A)$. The ths enhancer contains at least one high-affinity Snail repressor site that keeps the enhancer silent in the ventral mesoderm. $(B-D)$ Transgenic embryos that contain the $h t l$ enhancer attached to a lac $Z$ reporter gene. $L a c Z$ expression was detected by in situ hybridization. Staining is first detected in the ventral mesoderm during cellularization $(B)$ and persists in the mesoderm during formation of the ventral furrow $(C)$ and germ-band elongation $(D)$. $(F-H)$ Transgenic embryos that express the ths enhancer attached to a lac $Z$ reporter gene. Staining is detected in cellularizing embryos $(F)$ and persists in the ectoderm during the early stages of germ-band elongation $(G)$. After elongation expression is diminished $(H)$. $(I)$ Diagrams showing different patterns of expression generated by the Dorsal gradient. The circles represent cross-sections through early embryos. The Dorsal nuclear gradient present at stage 5 is depicted in the diagram on the top. High levels of Dorsal activate Htl and Dof/Hbr/Sms, whereas low levels activate Ths and Pyr, as well as Sog. These same low levels of nuclear Dorsal also repress Dpp and restrict its expression to the dorsal ectoderm. After invagination of the mesoderm (stage 7,8), Ths and Pyr activate Htl signaling and thereby control the spreading of the mesoderm toward the dorsal ectoderm (middle diagram). Mesoderm that reaches the dorsal ectoderm is induced by Dpp to form cardiac tissues (bottom diagram). 
enhancer was identified within the first intron of this gene as a cluster of two Dorsal-binding sites and a copy of a conserved sequence motif, RGGNCAG, which is seen in a variety of Dorsal target enhancers (Stathopoulos et al. 2002). When attached to the lacZ reporter gene, this cluster directs weak expression in the mesoderm of early embryos and tracheal pits of older embryos (data not shown).

These results provide evidence that $h t l$ and $d o f / h b r /$ sms are direct target genes of the Dorsal gradient that are induced in response to peak levels of nuclear Dorsal present in ventral regions of early embryos (Fig. 7I). The previously identified ths enhancer (previously called the Neu4 enhancer) contains three high-affinity Dorsalbinding sites and a Snail repressor site (Fig. 7E; Stathopoulos et al. 2002). The ths enhancer directs expression throughout the neurogenic ectoderm during early stages of gastrulation in response to lower levels of nuclear Dorsal (Fig. 7F-H). It is, therefore, possible to describe gastrulation as a series of discrete threshold readouts of the Dorsal gradient, as discussed below.

\section{Discussion}

Several lines of evidence suggest that Ths and Pyr correspond to ligands for the Htl FGF receptor. First, the two genes exhibit dynamic patterns of expression in tissues that influence the development of different mesoderm lineages, including the neurogenic ectoderm (early mesoderm spreading), muscle precursors (dorsal muscles, visceral muscles, and heart), hindgut (visceral musculature), and neuroblasts (patterning of the ventral oblique muscles). Second, mutant embryos that lack both $t h s^{+}$ and $\mathrm{pyr}^{+}$gene activity exhibit defects that are quite similar to those seen in htl mutants, including a delay in mesoderm spreading during gastrulation, a reduction in dorsal mesoderm lineages, the loss of pericardial and cardial cells, the absence of hindgut musculature, and disruptions in the ventral oblique muscles. Misexpression of Ths throughout the early mesoderm causes an expansion in the Eve expression pattern, consistent with expanded induction of pericardial and/or dorsal muscle founder cells. Finally, expression of activated $\mathrm{Htl}$ or Ths rescues the loss of dorsal mesoderm lineages in mutant embryos. Pyr and Ths might also activate the Htl receptor at later stages of the life cycle. For example, a recent microarray screen identified CG13194 (pyr) and CG12443 (ths) transcripts in the body wall muscle of wing imaginal disks, where $h t l$ is also expressed (Butler et al. 2003).

Previous genetic screens failed to identify ths and pyr, possibly because of overlap in the activities of the encoded proteins, which are closely related members of the FGF8/FGF17/FGF18 subfamily of FGF signaling molecules. Mutations in either gene alone might be insufficient to produce robust dorsal-ventral patterning defects, as seen for $h t l$ mutants. Indeed, two related FGF genes, FGF8 and FGF24, are required for the patterning of the posterior mesoderm in zebrafish embryos (Draper et al. 2003). A mutation in the FGF8 gene alone causes a relatively mild phenotype, but a severe loss of the pos- terior mesoderm is observed when FGF24 activity is also diminished. Similarly, we have shown that a small chromosome deficiency that removes both ths and pyr produces severe embryonic patterning defects.

It is conceivable that the spreading of the mesoderm across the internal surface of the neurogenic ectoderm is a simple manifestation of cell-cell contact. FGF signaling might cause each mesoderm cell to make maximal contact with the neurogenic ectoderm. According to this view, the Ths and Pyr ligands are permissive, and simply promote cell adhesion (e.g., Leptin 1999). An alternative view is that Ths and Pyr are spatially activated in a manner that promotes a temporal gradient of information that guides the movement of the mesoderm toward the dorsal ectoderm (e.g., Gabay et al. 1997). The expression of dpERK is consistent with an early requirement of FGF signaling acting permissively to activate $\mathrm{Htl}$ and allow the mesoderm to start spreading (Fig. 3G). Staining is first seen throughout the mesoderm that is in contact with the ectoderm during early phases of gastrulation when these individual mesoderm cells come into contact with the neurogenic ectoderm. Later, dpERK staining is restricted to the leading edge of the mesoderm as it spreads into the dorsal ectoderm. These data support a model in which the FGF ligands, Ths and/or Pyr, activate $\mathrm{Htl}$ in an instructive manner that guides the mesoderm during the later stage of spreading. The expression of ths and pyr is consistent with this model of early permissive and late instructive roles of the ligands in Htl activation (Fig. 7I; stage 7,8). Early, ths is expressed in a broad staining pattern, which might reflect a role in the promotion of initial contact between the mesoderm and neurogenic ectoderm. The restricted staining of both ths and pyr seen later may reflect a Ths/Pyr activity gradient emanating from increasingly more dorsal regions of the neurogenic ectoderm (e.g., Fig. 2G).

The combined ths and pyr expression profiles might produce a dynamic FGF activity gradient within the neurogenic ectoderm that guides the spreading of the mesoderm into the dorsal ectoderm. pyr expression is particularly dynamic, and rapidly lost in the neurogenic ectoderm, whereas ths expression is progressively lost first in ventral regions and then in more dorsal regions of the neurogenic ectoderm. In principle, this putative FGF gradient could provide a precise guidance cue for the coordinated spreading of the mesoderm into the dorsal ectoderm. However, it is also conceivable that the production of an FGF signaling gradient depends on posttranscriptional regulation, such as the translational regulation of mRNA expression or differential processing of FGF precursor proteins. For instance, the early embryonic enhancer isolated for ths does not support expression during germ-band elongation (Fig. $7 \mathrm{H}$ ) even though ths mRNA can be detected by in situ hybridization at this same stage (Fig. 2B,C). One interpretation of these results is that the ths mRNA is not synthesized during mesoderm migration. Differential degradation might help shape an FGF ligand activity gradient, as observed for FGF8 (Dubrulle and Pourquie 2004). In addition, negative regulators of signaling downstream of the $\mathrm{Htl}$ 
receptor could contribute to the production and sharpening of an FGF signaling activity gradient.

Ths and Pyr are related to FGF signaling molecules that control both cell movement and differentiation. For example, EGL-17 directs the movement of the sex myoblasts in the gonad and FGF8 is required for the migration of the mesoderm into the primitive streak of vertebrate embryos and later for heart development (Burdine et al. 1998; Sun et al. 1999; Reifers et al. 2000). We review the evidence that Ths and Pyr are required both for the spreading of the mesoderm along the internal surface of the neurogenic ectoderm during gastrulation, as well as the subsequent induction of the dorsal mesoderm to form pericardial tissues.

Evidence that neurogenic expression of Ths and Pyr is important for the orderly spreading of the mesoderm was obtained by misexpressing Ths (Fig. 6). Embryos misrepresenting Ths in the mesoderm exhibit a variety of defects including mild twisting of the germ band, abnormal patterning of the body wall muscles, and an expansion of cardiac tissues. The latter phenotype can be explained on the basis of expanded induction of dorsal mesoderm (there is at least a threefold increase in the number of Eve-expressing cells; see Fig. 6K,L, cf. J). A more uniform rescue phenotype was obtained when exogenous Ths products were expressed in the ectoderm using the 69Bgal4 transgene (Fig. 6N,O).

Once mesoderm spreading is complete, the leading edge of the mesoderm comes into contact with Dpp-expressing cells in the dorsal ectoderm. Dpp signaling might be sufficient for the activation of some of the target genes required for the patterning of the visceral mesoderm, such as tin and bap during stage 10 (StaehlingHampton et al. 1994; Frasch 1995). However, Dpp is insufficient for other inductive events such as the activation of tin and eve in different heart precursors. The loss of eve expression in ths; pyr and htl mutants does not appear to be due to a breakdown in mesoderm spreading. Although this spreading is delayed in the mutants, it does ultimately occur (see Fig. 3D,F). The late activation of the Htl receptor may be essential for the induction of eve expression and the specification of pericardial tissues. Previous studies suggest that Dpp works together with another signal that may be localized in the dorsal ectoderm. This second signal appears to trigger Ras signaling because the expression of a constitutively activated form of Ras causes expanded expression of eve (Halfon et al. 2000). Evidence that the second signal might be FGF stems from the analysis of a dominantnegative Htl receptor, which blocks the full expression of cardiac and pericardial gene markers after the mesoderm has spread (Michelson et al. 1998b). The present study considerably strengthens the case that FGF is the second signal that patterns the dorsal mesoderm. The misexpression of Ths in the mesoderm causes a substantial expansion in the dorsal mesoderm and the number of Eve-expressing cells. Moreover, ths and pyr are expressed in specific "spots" within the dorsal ectoderm that are adjacent to the internal mesoderm where eve is activated. Thus, the simplest interpretation of the results is that FGF signaling controls both the spreading and patterning of the dorsal mesoderm.

The spreading and subsequent subdivision of the mesoderm into distinct dorsal and ventral lineages can be viewed as direct readouts of the Dorsal gradient (summarized in Fig. 7I). The identification of mesoderm enhancers for htl and dof/hbr/smsf based on clustering of Dorsal-binding sites (and associated sequence motifs) suggests that these genes are directly activated by high levels of the Dorsal gradient. Htl-dependent signaling is triggered by Ths and Pyr, which are selectively expressed in the neurogenic ectoderm in response to low levels of the Dorsal gradient. After spreading, dorsal mesoderm cells comes into contact with Dpp-expressing cells in the dorsal ectoderm, and are thereby induced to form dorsal lineages such as cardiac tissues. The same low levels of the Dorsal gradient that activate ths and pyr also activate sog expression and repress $d p p$. The Sog inhibitor ensures that Dpp signaling is restricted to the dorsal ectoderm. Thus, the differential regulation of $\mathrm{Htl}$ and its ligands determines the precise limits of mesoderm-ectoderm germ-layer interactions during gastrulation.

\section{Materials and methods}

\section{Drosophila stocks and genetic crosses}

All crosses and misexpression experiments were performed at $25^{\circ} \mathrm{C}$. Wild-type flies refer to $y w$ mutants. Df(2R)BSC25 was isolated as a P-transposase-induced male recombination event involving $\mathrm{P}\{\mathrm{lacW}\} \mathrm{wal}^{\mathrm{k} 14026}$ and $\mathrm{P}\{\mathrm{PZ}\} \mathrm{Cct} 5^{06444}$ (Bloomington Stock Center). We conducted inverse PCR to determine the breakpoints of the deficiency. The $5^{\prime}$-end of PfbrlacW|wal ${ }^{\mathrm{k} 14026}$ is now present in the $5^{\prime}-\mathrm{UTR}$ of the Ef $1 \alpha 48 \mathrm{D}$ gene, resulting in a deletion of roughly 200,000 bases upstream of wal.

Standard crosses were performed for all genetic manipulations. Flies of the genotype twi-Gal4; $D f(2 R) B S C 25 / C y O \mathrm{ftz}$ $l a c Z$ were mated to those of the genotype $D f(2 R) B S C 25 / C y O$ ftz-lacZ; UAS-activated(act)Htl/+, Df(2R)BSC25/CyO ftz-lacZ; UAS-Ht1/+, or Df(2R)BSC25/CyO ftz-lacZ; UAS-ths/+. Embryos examined were of the genotype twi-Gal4; $D f(2 R) B S C 25 /$ $D f(2 R) B S C 25$; UAS-actHtl, UAS-Ht1, or UAS-ths. Those expected to contain twiGal4+UAS-actHtl or twiGal4+UAS-ths exhibit Eve-expressing cells. None of the mutant embryos containing twiGal4+UAS-Htl exhibited Eve expression. For the UAS-Thisbe misexpression, twi-Gal4 females were crossed to males containing the UAS-Thisbe transgene on either the $\mathrm{X}$ chromosome or Chromosome 2.

\section{RACE analysis and molecular biology}

The GeneRacer kit (Invitrogen) was used to isolate $5^{\prime}$ and $3^{\prime}$ sequences from both CG12443/Neu4 and CG13194. RNA was isolated from embryos aged $0-4 \mathrm{~h}$ or $4-8 \mathrm{~h}$ and reverse-transcribed to generate cDNA using both random primers and poly(dT). Gene-specific primers used to determine the $5^{\prime}$ - and $3^{\prime}$-ends were 5'-CCCTTGAAACCCACACGCAGCACTGG AT-3' and 5'-GCCACGCCCTACCACCAGCTGACCTATG-3' for Neu4 and 5'-TTCCAACCAGTCTGCCGTGATGGAAGC A-3' and 5'-GCTTGGCAATTTGCATGTCACTGTGG-3' for CG13194.

A full-length thisbe cDNA was isolated from reverse-transcribed RNA isolated from early embryos. Primers flanking the ATG and TAA codons predicted by the RACE analysis were used to isolate an $\sim 2.0-\mathrm{kb}$ transcript containing the entire ORF. 
thsbe cDNA was inserted into the BglII-XbaI sites of pUAST, and this resulting P-element construct was injected into flies to generate transformants.

Fragments representing the $h t 1$ and $d o f / h b r / s m s$ enhancers were isolated from $y w$ genomic DNA using the following primers: 5'-AAAGTCGAGTTGCCAAATTGCCAGG-3' and 5'-GGCGATTTTGGACGGCACAGGAA-3' for $h t l$ and 5'GAAAGATGAGGGACACGGCTTCAAA- ${ }^{\prime}$ and $5^{\prime}$-CGAGAG AGCGAGCAGGAGCATCTAC-3' for $\mathrm{dof} / \mathrm{hbr} / \mathrm{sms}$ and cloned into the EcoRI site of the P-element injection vector -42evelacZCasper (Stathopoulos et al. 2002). Five of five lines showed expected expression for htl-lacZ, whereas only two of six showed expected expression for dof/hbr/sms-lacZ.

\section{Whole-mount in situ hybridization and antibody staining}

Appropriately staged embryos were fixed and hybridized with digoxigenin-UTP antisense RNA probes as described previously (Jiang et al. 1991; Lehmann and Tautz 1994). Probes to pyramus, thisbe, tinman, and bagpipe were made from 1-2-kb PCR products amplified from coding sequences of these genes. A probe to twist was made using the Twist cDNA (generously provided by M. Leptin, University of Koeln, Koeln, Germany). Generation of a probe to lacZ has been previously described (Jiang et al. 1991).

Antibodies were used at various dilutions with the Vectastain antibody staining kit (Vector) to examine protein levels in embryos: anti-Twist rabbit antibody (generously provided by $\mathrm{S}$. Roth, Cologne University, Cologne, Germany) at 1:3000, antidpERK monoclonal antibody at 1:100 (SIGMA), anti-Eve rabbit antibody at 1:6000, anti- $\beta$-gal rabbit antibody (Cappel) at 1:250, anti- $\beta$-gal mouse antibody (Promega) at 1:2000, and anti-Mef2 rabbit antibody (generously provided by Hanh Nguyen, Albert Einstein College of Medicine, Bronx, NY) at 1:500. Primary antibody incubations were performed overnight and secondary antibody incubations for $3 \mathrm{~h}$ at room temperature. Embryos were embedded in plastic and sectioned as previously described (Azpiazu and Frasch 1993).

\section{Protein alignment and phylogenetic inference}

FGF protein sequences used in alignment and phylogenetic reconstruction were gathered from GenBank or inferred from genomic sequence using GENESCAN (Burge and Karlin 1997) and FGENESH. Alignments were performed using both CLUSTALX (Thompson et al. 1997) and MAFFT (Katoh et al. 2002). Phylogenetic relationships were inferred using neighbor joining (NJ) and maximum likelihood (ML) from a 169-amino acid alignment containing only the FGF trefoil domain. Support for NJ topologies was evaluated using bootstrap analysis. Support for maximum likelihood trees used quartet puzzling reliability values from 10,000 puzzling steps. The NJ inference of tree topology and the subsequent bootstrap analysis were implemented in CLUSTALX (Thompson et al. 1997). The quartet puzzling maximum likelihood analysis was performed with TREE-PUZZLE (Strimmer and von Haeseler 1997). The ML model of evolution allowed for among-site rate heterogeneity with eight g-distributed rate categories. Accession numbers for sequences are as follows: D.mel Thisbe (accession no. AY553965), D.pse Thisbe (Baylor Contig 4548), A.gam Thisbe (aaab01008816.1), D.mel Pyramus (accession no. AY553964), D.pse Pyramus (Baylor Contig 2718), Worm egl-17 (AAD00574), Human FGF-8 (NP_034335), Human FGF-17 (NP_003858), Human FGF-18 (NP_387498), Zebrafish FGF-24 (NP_878291), D.mel Branchless (NP_732452), NPHV FGF (NP_047439),Worm let-756 (Q11184), Human FGF-7 (NP_002000), and Human FGF-10 (O15520).

\section{Acknowledgments}

We thank S. Roth and H. Nguyen for providing antibodies and A. Michelson and the Bloomington Stock Center for providing fly stocks. We are grateful to Kevin Cook for information regarding the BSC25 deficiency, Yutaka Satou for help with the FGF classification, and Kate Senger for comments on the manuscript. This work was funded from grants from the NIH: GM46638 to M.L., HD30832 to M.F., and GM20352 to A.S.

The publication costs of this article were defrayed in part by payment of page charges. This article must therefore be hereby marked "advertisement" in accordance with 18 USC section 1734 solely to indicate this fact.

\section{References}

Ahmad, S.M. and Baker, B.S. 2002. Sex-specific deployment of FGF signaling in Drosophila recruits mesodermal cells into the male genital imaginal disc. Cell 109: 651-661.

Anderson, K.V., Jurgens, G., and Nusslein-Volhard, C. 1985. Establishment of dorsal-ventral polarity in the Drosophila embryo: Genetic studies on the role of the Toll gene product. Cell 42: 779-789.

Azpiazu, N. and Frasch, M. 1993. tinman and bagpipe: Two homeo box genes that determine cell fates in the dorsal mesoderm of Drosophila. Genes \& Dev. 7: 1325-1340.

Bateman, A., Birney, E., Cerruti, L., Durbin, R., Etwiller, L., Eddy, S.R., Griffiths-Jones, S., Howe, K.L., Marshall, M., and Sonnhammer, E.L. 2002. The Pfam protein family database. Nucleic Acids Res. 30: 276-280.

Beiman, M., Shilo, B.Z., and Volk, T. 1996. Heartless, a Drosophila FGF receptor homolog, is essential for cell migration and establishment of several mesodermal lineages. Genes \& Dev. 10: 2993-3002.

Bodmer, R. 1993. The gene tinman is required for specification of the heart and visceral muscles in Drosophila. Development 118: 719-729.

Bour, B.A., O’Brien, M.A., Lockwood, W.L., Goldstein, E.S., Bodmer, R., Taghert, P.H., Abmayr, S.M., and Nguyen, H.T. 1995. Drosophila MEF2, a transcription factor that is essential for myogenesis. Genes \& Dev. 9: 730-741.

Burdine, R.D., Chen, E.B., Kwok, S.F., and Stern, M.J. 1997. egl-17 encodes an invertebrate fibroblast growth factor family member required specifically for sex myoblast migration in Caenorhabditis elegans. Proc. Nat1. Acad. Sci. 94: 24332437.

Burdine, R.D., Branda, C.S., and Stern, M.J. 1998. EGL-17(FGF) expression coordinates the attraction of the migrating sex myoblasts with vulval induction in C. elegans. Development 125: 1083-1093.

Burge, C. and Karlin, S. 1997. Prediction of complete gene structures in human genomic DNA. J. Mol. Biol. 268: 78-94.

Butler, M.J., Jacobsen, T.L., Cain, D.M., Jarman, M.G., Hubank, M., Whittle, J.R., Phillips, R., and Simcox, A. 2003. Discovery of genes with highly restricted expression patterns in the Drosophila wing disc using DNA oligonucleotide microarrays. Development 130: 659-670.

Carmena, A., Gisselbrecht, S., Harrison, J., Jimenez, F., and Michelson, A.M. 1998. Combinatorial signaling codes for the progressive determination of cell fates in the Drosophila embryonic mesoderm. Genes \& Dev. 12: 3910-3922.

Draper, B.W., Stock, D.W., and Kimmel, C.B. 2003. Zebrafish fgf 24 functions with fgf8 to promote posterior mesodermal development. Development 130: 4639-4654.

Dubrulle, J. and Pourquie, O. 2004. fgf8 mRNA decay establishes a gradient that couples axial elongation to patterning 
in the vertebrate embryo. Nature 427: 419-422.

Frasch, M. 1995. Induction of visceral and cardiac mesoderm by ectodermal Dpp in the early Drosophila embryo. Nature 374: 464-467.

Gabay, L., Seger, R., and Shilo, B.Z. 1997. MAP kinase in situ activation atlas during Drosophila embryogenesis. Development 124: 3535-3541.

Gajewski, K., Fossett, N., Molkentin, J.D., and Schulz, R.A. 1999. The zinc finger proteins Pannier and GATA4 function as cardiogenic factors in Drosophila. Development 126: $5679-5688$

Gisselbrecht, S., Skeath, J.B., Doe, C.Q., and Michelson, A.M. 1996. heartless encodes a fibroblast growth factor receptor (DFR1/DFGF-R2) involved in the directional migration of early mesodermal cells in the Drosophila embryo. Genes \& Dev. 10: 3003-3017.

Greig, S. and Akam, M. 1993. Homeotic genes autonomously specify one aspect of pattern in the Drosophila mesoderm. Nature 362: 630-632.

Halfon, M.S., Carmena, A., Gisselbrecht, S., Sackerson, C.M., Jimenez, F., Baylies, M.K., and Michelson, A.M. 2000. Ras pathway specificity is determined by the integration of multiple signal-activated and tissue-restricted transcription factors. Cell 103: 63-74.

Harvey, N.L., Daish, T., Mills, K., Dorstyn, L., Quinn, L.M., Read, S.H., Richardson, H., and Kumar, S. 2001. Characterization of the Drosophila caspase, DAMM. J. Biol. Chem. 276: 25342-25350.

Imam, F., Sutherland, D., Huang, W., and Krasnow, M.A. 1999. stumps, a Drosophila gene required for fibroblast growth factor (FGF)-directed migrations of tracheal and mesodermal cells. Genetics 152: 307-318.

Jiang, J., Kosman, D., Ip, Y.T., and Levine, M. 1991. The dorsal morphogen gradient regulates the mesoderm determinant twist in early Drosophila embryos. Genes \& Dev. 5: 18811891.

Katoh, K., Misawa, K., Kuma, K., and Miyata, T. 2002. MAFFT: A novel method for rapid multiple sequence alignment based on fast Fourier transform. Nucleic Acids Res. 30: 3059-3066.

Lehmann, R. and Tautz, D. 1994. In situ hybridization to RNA. Methods Cell Biol. 44: 575-598.

Leptin, M. 1999. Gastrulation in Drosophila: The logic and the cellular mechanisms. EMBO J. 18: 3187-3192.

Lilly, B., Zhao, B., Ranganayakulu, G., Paterson, B.M., Schultz, R.A., and Olson, E.N. 1995. Requirement of MADS domain transcription factor D-MEF2 for muscle formation in Drosophila. Science 267: 688-693.

Liu, X., Kiss, I., and Lengyel, J.A. 1999. Identification of genes controlling Malpighian tubule and other epithelial morphogenesis in Drosophila melanogaster. Genetics 151: 685-695.

Maruoka, Y., Ohbayashi, N., Hoshikawa, M., Itoh, N., Hogan, B.L., and Furuta, Y. 1998. Comparison of the expression of three highly related genes, Fgf8, Fgf17 and Fgf18, in the mouse embryo. Mech. Dev. 74: 175-177.

Michelson, A.M., Gisselbrecht, S., Buff, E., and Skeath, J.B. 1998a. Heartbroken is a specific downstream mediator of FGF receptor signalling in Drosophila. Development 125: 4379-4389.

Michelson, A.M., Gisselbrecht, S., Zhou, Y., Baek, K.H., and Buff, E.M. 1998b. Dual functions of the heartless fibroblast growth factor receptor in development of the Drosophila embryonic mesoderm. Dev. Genet. 22: 212-229.

Nielsen, H., Engelbrecht, J., Brunak, S., and von Heijne, G. 1997. A neural network method for identification of prokaryotic and eukaryotic signal peptides and prediction of their cleav- age sites. Int. J. Neural Syst. 8: 581-599.

Ornitz, D.M. 2000. FGFs, heparan sulfate and FGFRs: Complex interactions essential for development. Bioessays 22: 108112.

Reifers, F., Walsh, E.C., Leger, S., Stainier, D.Y.R., and Brand, M. 2000. Induction and differentiation of the zebrafish heart requires fibroblast growth factor 8 (fgf8/acerebellar). Development 127: 225-235.

Roubin, R., Naert, K., Popovici, C., Vatcher, G., Coulier, F., Thierry-Mieg, J., Pontarotti, P., Birnbaum, D., Baillie, D., and Thierry-Mieg, D. 1999. let-756, a C. elegans fgf essential for worm development. Oncogene 18: 6741-6747.

San Martin, B. and Bate, M. 2001. Hindgut visceral mesoderm requires an ectodermal template for normal development in Drosophila. Development 128: 233-242.

Sato, M. and Kornberg, T.B. 2002. FGF is an essential mitogen and chemoattractant for the air sacs of the Drosophila tracheal system. Dev. Cell 3: 195-207.

Shishido, E., Ono, N., Kojima, T., and Saigo, K. 1997. Requirements of DFR1/Heartless, a mesoderm-specific Drosophila FGF-receptor, for the formation of heart, visceral and somatic muscles, and ensheathing of longitudinal axon tracts in CNS. Development 124: 2119-2128.

Simpson, P. 1983. Maternal-zygotic gene interactions during formation of the dorsoventral pattern in Drosophila embryos. Genetics 105: 615-632.

Staehling-Hampton, K., Hoffmann, F.M., Baylies, M.K., Rushton, E., and Bate, M. 1994. dpp induces mesodermal gene expression in Drosophila. Nature 372: 783-786.

Stathopoulos, A., Van Drenth, M., Erives, A., Markstein, M., and Levine, M. 2002. Whole-genome analysis of dorsal-ventral patterning in the Drosophila embryo. Cell 111: 687-701.

Strimmer, K. and von Haeseler, A. 1997. Likelihood-mapping: A simple method to visualize phylogenetic content of a sequence alignment. Proc. Nat1. Acad. Sci. 94: 6815-6819.

Sun, X., Meyers, E.N., Lewandoski, M., and Martin, G.R. 1999. Targeted disruption of Fgf8 causes failure of cell migration in the gastrulating mouse embryo. Genes \& Dev. 13: 18341846.

Sutherland, D., Samakovlis, C., and Krasnow, M.A. 1996. branchless encodes a Drosophila FGF homolog that controls tracheal cell migration and the pattern of branching. Cell 87: 1091-1101.

Szebenyi, G. and Fallon, J.F. 1999. Fibroblast growth factors as multifunctional signaling factors. Int. Rev. Cytol. 185: 45106

Thompson, J.D., Gibson, T.J., Plewniak, F., Jeanmougin, F., and Higgins, D.G. 1997. The CLUSTAL_X windows interface: Flexible strategies for multiple sequence alignment aided by quality analysis tools. Nucleic Acids Res. 25: 4876-4882.

Tickle, C. and Munsterberg, A. 2001. Vertebrate limb development-The early stages in chick and mouse. Curr. Opin. Genet. Dev. 11: 476-481.

Vincent, S., Wilson, R., Coelho, C., Affolter, M., and Leptin, M. 1998. The Drosophila protein Dof is specifically required for FGF signaling. Mol. Cell 2: 515-525.

Wilson, R. and Leptin, M. 2000. Fibroblast growth factor receptor-dependent morphogenesis of the Drosophila mesoderm. Philos. Trans. R Soc. Lond. B Biol. Sci. 355: 891-895. 


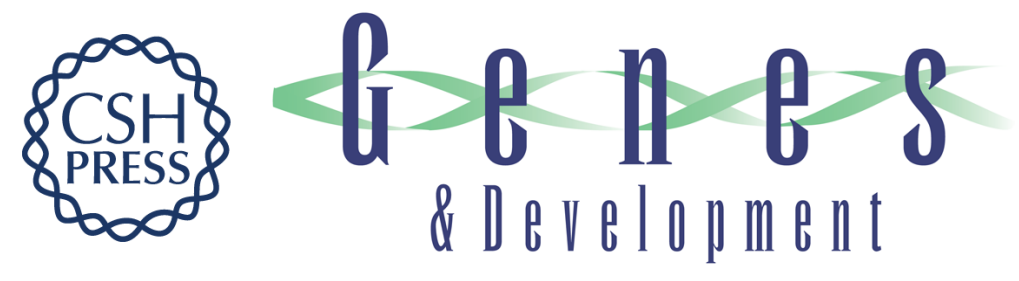

\section{pyramus and thisbe: FGF genes that pattern the mesoderm of Drosophila embryos}

Angelike Stathopoulos, Bergin Tam, Matthew Ronshaugen, et al.

Genes Dev. 2004, 18:

Access the most recent version at doi:10.1101/gad.1166404

References This article cites 49 articles, 25 of which can be accessed free at: http://genesdev.cshlp.org/content/18/6/687.full.html\#ref-list-1

License

Email Alerting Receive free email alerts when new articles cite this article - sign up in the box at the top Service right corner of the article or click here.

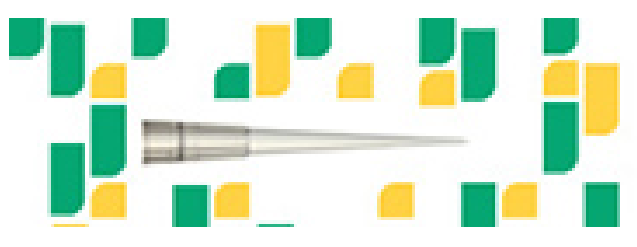

Focused on your science. 